\title{
Thermal Cyclodehydrogenations to Form 6-Membered Rings: Cyclizations of [5]Helicenes
}

\author{
Xiang Xue and Lawrence T. Scott ${ }^{\star}$ \\ Merkert Chemistry Center, Department of Chemistry, Boston College, Chestnut Hill, \\ Massachusetts 02467-3860
}

Lawrence.scott@bc.edu

Received Date (will be automatically inserted after manuscript is accepted)

Experimental procedures

Synthesis and NMR spectra of naphtho[1,2-g]chrysene (6)

page $\mathrm{S} 2$

Flash vacuum pyrolysis details

page S5

${ }^{1} \mathrm{H}$ NMR spectrum of the crude product from FVP of $\mathbf{1}$ at $1000{ }^{\circ} \mathrm{C}$

page S6

${ }^{1} \mathrm{H}$ NMR spectrum of the crude product mixture from FVP of 6 at $1000{ }^{\circ} \mathrm{C}$

page $\mathrm{S} 7$

Scheme S1. Proposed mechanism for formation of the minor product (10)

page $\mathrm{S} 8$

Scheme S2. Homodesmic energy comparisons between the parent [5]helicene (1) and the benzo[5]helicene

(6) for the three cyclodehydrogenation pathways illustrated in Scheme 2 (UB3LYP/6-31G*)

page $\mathrm{S} 9$

Calculated XYZ coordinates and energies from UB3LYP/6-31G* geometry optimizations on $\mathbf{1}, \mathbf{3}, \mathbf{4}, \mathbf{5}, \mathbf{6}$,

$\mathbf{7}$, and the benzannulated derivatives of $\mathbf{3}$ and $\mathbf{4}$

page $\mathrm{S} 10$ 


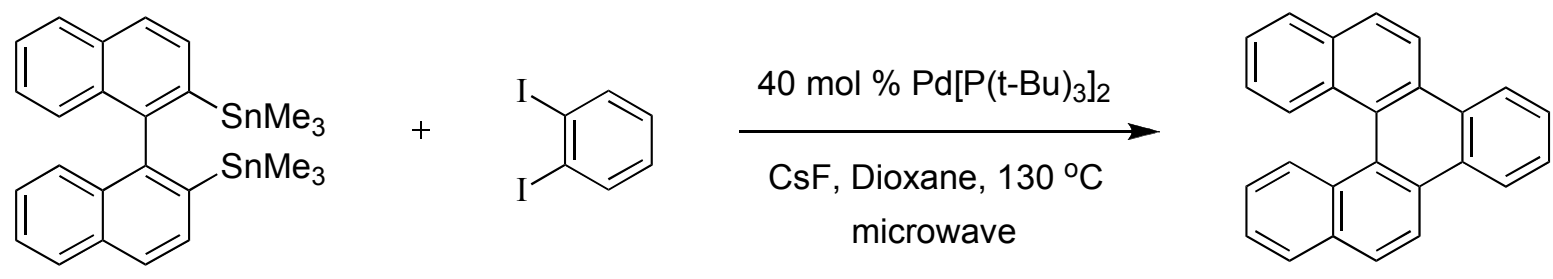

\section{Synthesis of naphtho[1,2-g]chrysene (6).}

2,2'-Bis(trimethylstannyl)-1,1'-binaphthyl ${ }^{1}(58 \mathrm{mg}, 0.10 \mathrm{mmol}), \operatorname{Pd}\left[\mathrm{P}(t-\mathrm{Bu})_{3}\right]_{2}(21 \mathrm{mg}, 0.04 \mathrm{mmol})$ and cesium fluoride ( $76 \mathrm{mg}, 0.50 \mathrm{mmol}$ ) were added into an oven-dried $10 \mathrm{~mL}$ glass reaction vial (pressurerated up to 35 Bar ( 515 PSI)) equipped with a Teflon coated magnetic stirring bar. The vial was then sealed with a PTFE-Silicon septum in an Intellivent Cap and purged repeatedly with argon. Anhydrous 1,4-dioxane $(1 \mathrm{~mL})$ and 1, 2-diiodobenzene $(13 \mu \mathrm{L}, 0.10 \mathrm{mmol})$ were added to this solid mixture, and the vial was then irradiated with microwaves in a CEM Discover Microwave Unit, at $130{ }^{\circ} \mathrm{C}$ for $45 \mathrm{~min}$ with a $150 \mathrm{~W}$ power max. The solvent was removed under reduced pressure, and the crude residue was dissolved in $50 \mathrm{~mL}$ of methylene chloride. The organic solution was then passed through a silica gel plug, washed with $10 \% \mathrm{HCl}$, water and brine, dried with $\mathrm{MgSO}_{4}$, filtered and concentrated to dryness under reduced pressure. Purification of the crude product by silica gel chromatography using hexanes as the eluent gave an off-white solid $(27 \mathrm{mg}, 82 \%)$, the spectral data of which matched those reported by Tinnemans et al. ${ }^{2}$

\footnotetext{
${ }^{1}$ Hoshi, T.; Shionoiri, H.; Katano, M.; Suzuki, T.; Ando, M.; Hagiwara, H. Chem. Lett. 2002, 600-601.

${ }^{2}$ Tinnemans, A. H. A.; Laarhoven, W. H.; Sharafi-Ozeri, S.; Muszkat, K. A. Recl. Trav. Chim. Pays-Bas 1975, 94, 239.
} 


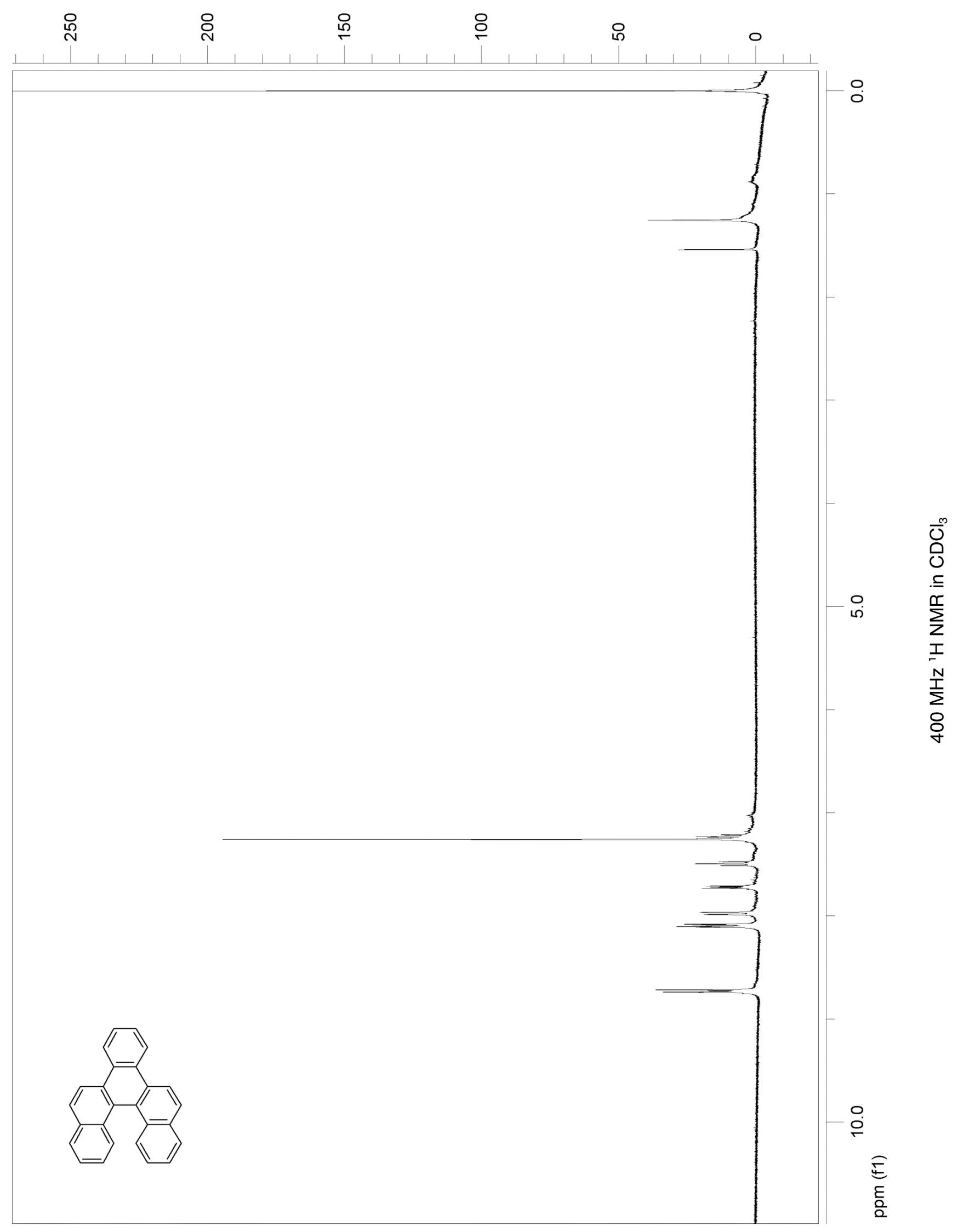




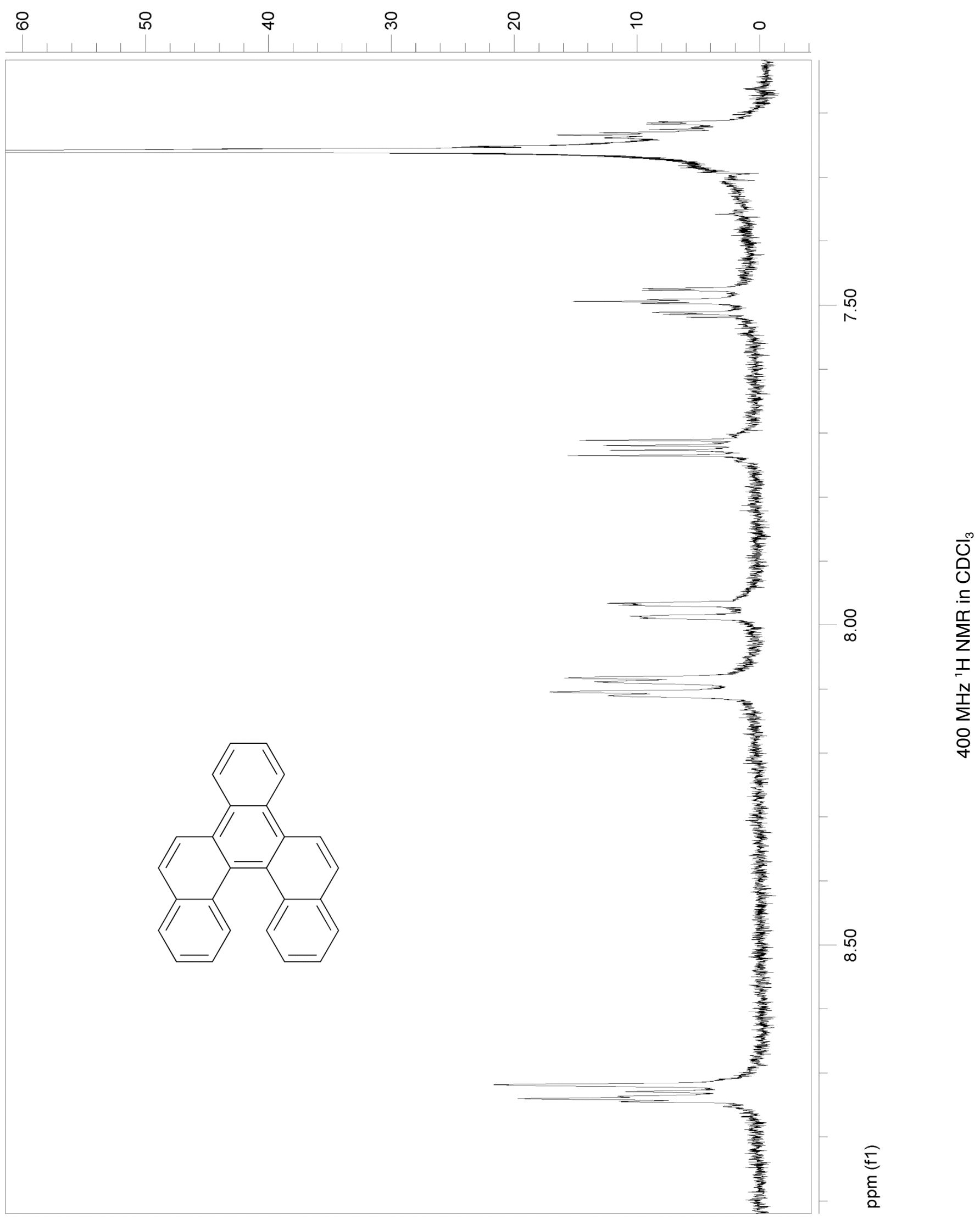




\section{Flash vacuum pyrolyses of [5]helicene (1) and benzo[5]helicene (6).}

Fifty milligram samples of [5]helicene (1) and benzo[5]helicene (6) were slowly sublimed into a gentle stream of nitrogen carrier gas under vacuum $(0.4-1.0 \mathrm{mmHg})$ in a flash vacuum pyrolysis apparatus that has been described elsewhere in detail. ${ }^{3}$ The hot zone was maintained at temperatures ranging from $700-1000{ }^{\circ} \mathrm{C}$, and the products were collected in a trap cooled by liquid nitrogen. The crude pyrolysis

product mixtures were dissolved in dichloromethane and analyzed by ${ }^{1} \mathrm{H}$ NMR spectroscopy before any separation was attempted by chromatography. The mass of the recovered material relative to the mass of the starting helicene varied from $66-96 \%$. Figures S1 and S2 show the ${ }^{1} \mathrm{H}$ NMR spectra of the products from FVP of [5]helicene (1) and benzo[5]helicene (6), respectively, at $1000{ }^{\circ} \mathrm{C}$. The former shows only a single product (2) and no trace of uncyclized helicene. In Figure S2, signals can be seen for residual starting material (6), the expected product (8), and the unexpected product (10). ${ }^{1} \mathrm{H}$ NMR spectra of the products from FVP of $\mathbf{1}$ and 6 at $850^{\circ} \mathrm{C}$ are reproduced in Figure 2 of the paper.

\footnotetext{
${ }^{3}$ Tsefrikas, V. M.; Scott, L. T. Chem. Rev. 2006, 106, 4868.
} 
<smiles>c1ccc2c(c1)ccc1ccc3ccc4ccccc4c3c12</smiles>

1

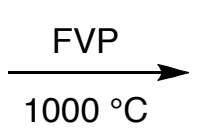

$1000^{\circ} \mathrm{C}$<smiles></smiles>

2 $\left(\begin{array}{c}\text { no starting } \\ \text { material left }\end{array}\right)$

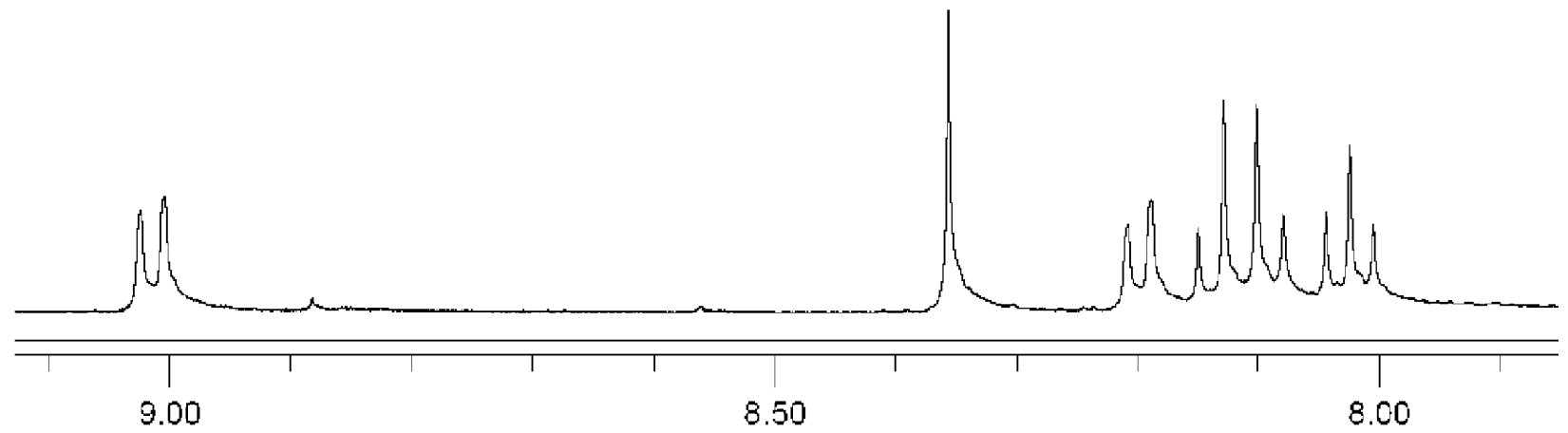

Figure S1. ${ }^{1} \mathrm{H}$ NMR $\left(400 \mathrm{MHz}, \mathrm{CDCl}_{3}\right.$ ) of product from FVP of [5]helicene (1) at $1000{ }^{\circ} \mathrm{C}$. 
<smiles></smiles>

6

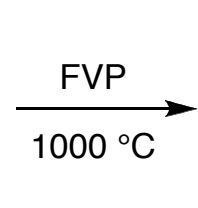

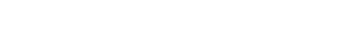<smiles></smiles>

8

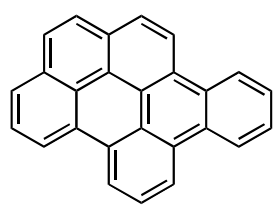

10

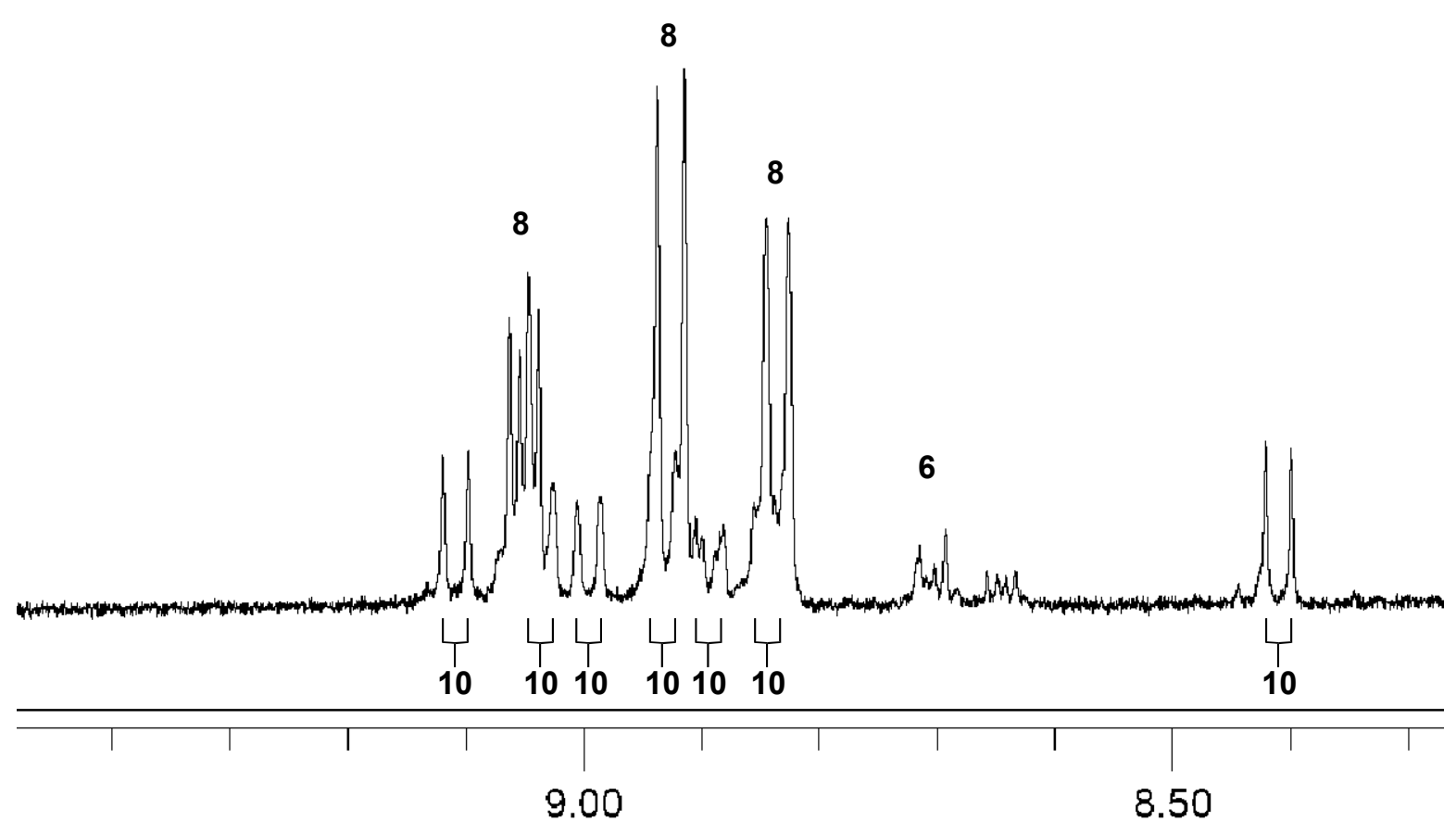

Figure S2. ${ }^{1} \mathrm{H}$ NMR $\left(400 \mathrm{MHz}, \mathrm{CDCl}_{3}\right)$ of products from $\mathrm{FVP}$ of benzo[5]helicene $(\mathbf{6})$ at $1000{ }^{\circ} \mathrm{C}$. 
Thermal Cyclodehydrogenations of [5]Helicenes

Xe and Scott<smiles>C1=Cc2c(c3c4ccccc4ccc3c3ccccc23)C=Cc2ccccc21</smiles>

6
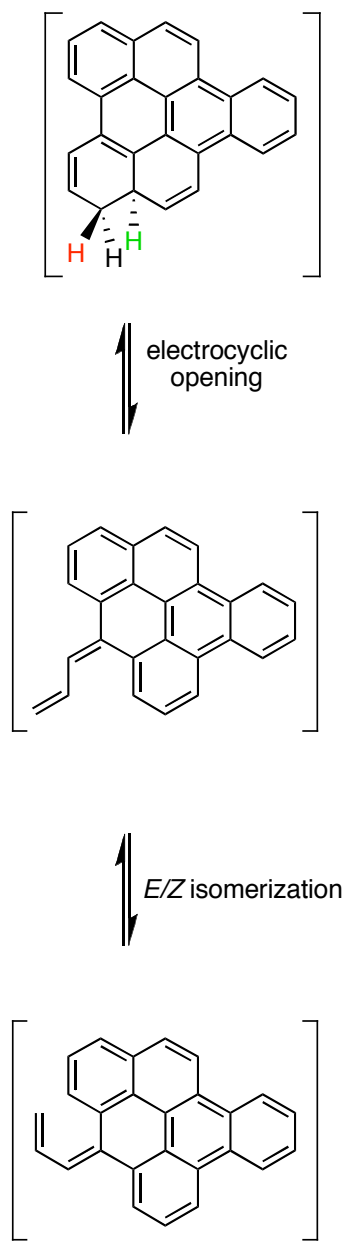

Scheme S1. Proposed mechanism for formation of the minor product (10).
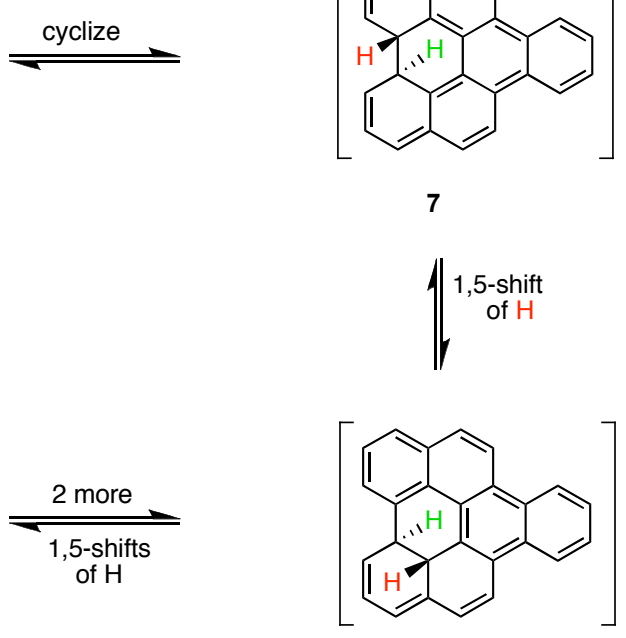

electrocyclic closure

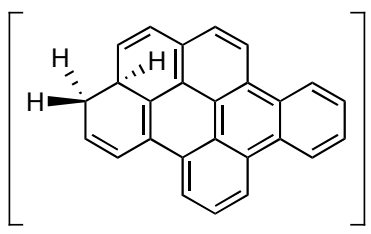

7

$\mid \begin{gathered}1,5-\text { shift } \\ \text { of } \mathrm{H}\end{gathered}$

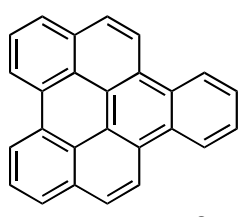

8
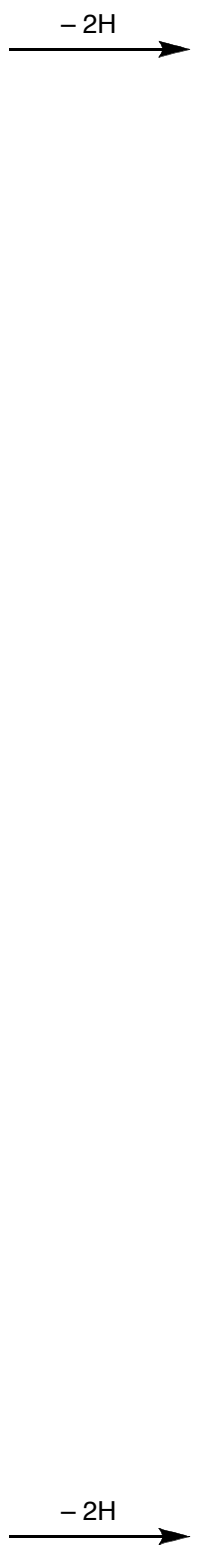

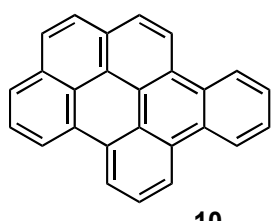

10

- SB - 
Scheme S2. Homodesmic energy comparisons between the parent [5]helicene (1) and the benzo[5]helicene (6) for the three cyclodehydrogenation pathways illustrated in Scheme 2 (UB3LYP/6-31G*)<smiles>c1ccc2c(c1)ccc1ccc3ccc4ccccc4c3c12</smiles>

1<smiles></smiles>

benzo-3
$\mathrm{E}_{\mathrm{rxn}}=-0.2 \mathrm{kcal} / \mathrm{mol}$<smiles>C1CC1</smiles><smiles>c1ccc2c(c1)ccc1ccc3ccc4ccccc4c3c12</smiles>

1<smiles>[CH2+]C1[CH]c2c(ccc3c4ccccc4c4ccc5ccccc5c4c23)C=C1</smiles>

benzo-4
$E_{\mathrm{rxn}}=+0.2 \mathrm{kcal} / \mathrm{mol}$<smiles></smiles>

4<smiles>c1ccc2c(c1)ccc1ccc3ccc4ccccc4c3c12</smiles>

3<smiles>C1=Cc2c(c3c4ccccc4ccc3c3ccccc23)C=Cc2ccccc21</smiles>

6<smiles>c1ccc2c(c1)ccc1ccc3ccc4ccccc4c3c12</smiles>

1

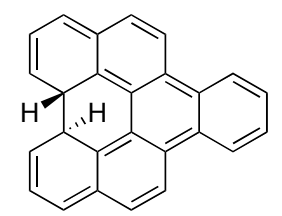

7
$E_{r x n}=-4.9 \mathrm{kcal} / \mathrm{mol}$<smiles>C1=C[C@@H]2C=Cc3ccc4ccc5ccc6c(c5c4c32)C(=C1)C=C6</smiles>

5<smiles>c1ccc2c(c1)ccc1c3ccccc3c3ccc4ccccc4c3c21</smiles>

6<smiles>c1ccc2c(c1)ccc1c3ccccc3c3ccc4ccccc4c3c21</smiles>

6 
Calculated DFT (UB3LYP/6-31G*) energy and X,Y,Z-coordinates for [5]helicene 1

\begin{tabular}{|c|c|c|c|c|}
\hline & & Coor & nates & roms ) \\
\hline & $\mathrm{OM}$ & $\mathrm{X}$ & $\mathrm{Y}$ & $\mathrm{Z}$ \\
\hline 1 & $\mathrm{C}$ & 0.724921 & -0.031881 & 0.856720 \\
\hline 2 & $\mathrm{H}$ & -1.188836 & 0.355971 & 4.252307 \\
\hline 3 & $\mathrm{C}$ & -0.724921 & 0.031881 & 0.856720 \\
\hline 4 & $\mathrm{C}$ & -1.377037 & 0.285829 & 2.095887 \\
\hline 5 & C & 1.377037 & -0.285829 & 2.095887 \\
\hline 6 & C & -1.141013 & -0.941720 & -1.446124 \\
\hline 7 & $\mathrm{C}$ & 0.656086 & -0.188078 & 3.319652 \\
\hline 8 & $\mathrm{C}$ & 2.772182 & -0.603012 & 2.126856 \\
\hline 9 & $\mathrm{C}$ & -0.656086 & 0.188078 & 3.319652 \\
\hline 10 & $\mathrm{H}$ & 1.188836 & -0.355971 & 4.252307 \\
\hline 11 & $\mathrm{C}$ & -1.582385 & -0.238289 & -0.294870 \\
\hline 12 & C & -2.772182 & 0.603012 & 2.126856 \\
\hline 13 & $\mathrm{C}$ & 3.529521 & -0.575295 & 0.996191 \\
\hline 14 & $\mathrm{H}$ & 3.213802 & -0.870799 & 3.083740 \\
\hline 15 & $\mathrm{C}$ & 2.973156 & -0.091007 & -0.228392 \\
\hline 16 & $\mathrm{H}$ & 4.583681 & -0.840353 & 1.023626 \\
\hline 17 & $\mathrm{C}$ & 1.582385 & 0.238289 & -0.294870 \\
\hline 18 & C & 3.809668 & 0.149277 & -1.346341 \\
\hline 19 & $\mathrm{C}$ & -3.809668 & -0.149277 & -1.346341 \\
\hline 20 & $\mathrm{C}$ & 1.141013 & 0.941720 & -1.446124 \\
\hline 21 & $\mathrm{C}$ & -3.529521 & 0.575295 & 0.996191 \\
\hline 22 & $\mathrm{H}$ & -3.213802 & 0.870799 & 3.083740 \\
\hline 23 & $\mathrm{C}$ & -2.973156 & 0.091007 & -0.228392 \\
\hline 24 & $\mathrm{H}$ & -4.583681 & 0.840353 & 1.023626 \\
\hline 25 & $\mathrm{C}$ & 3.327466 & 0.773122 & -2.476693 \\
\hline 26 & $\mathrm{H}$ & 4.856416 & -0.138496 & -1.279546 \\
\hline 27 & $\mathrm{C}$ & 1.985966 & 1.202125 & -2.507039 \\
\hline 28 & $\mathrm{H}$ & 3.982524 & 0.964636 & -3.322233 \\
\hline 29 & C & -1.985966 & -1.202125 & -2.507039 \\
\hline 30 & $\mathrm{H}$ & 1.612095 & 1.752336 & -3.366239 \\
\hline 31 & $\mathrm{H}$ & -4.856416 & 0.138496 & -1.279546 \\
\hline 32 & $\mathrm{C}$ & -3.327466 & -0.773122 & -2.476693 \\
\hline 33 & $\mathrm{H}$ & -3.982524 & -0.964636 & -3.322233 \\
\hline 34 & $\mathrm{H}$ & -1.612095 & -1.752336 & -3.366239 \\
\hline 35 & $\mathrm{H}$ & -0.121582 & -1.303173 & -1.487501 \\
\hline 36 & $\mathrm{H}$ & 0.121582 & 1.303173 & -1.487501 \\
\hline
\end{tabular}

Energy is $\quad-846.806952214$ 
Calculated DFT (UB3LYP/6-31G*) energy and X,Y,Z-coordinates for radical $\mathbf{3}^{\S}$

\begin{tabular}{|c|c|c|c|c|}
\hline & & \multicolumn{3}{|c|}{ Coordinates (Angstroms) } \\
\hline & ATOM & $\mathrm{x}$ & $\mathrm{Y}$ & $\mathrm{z}$ \\
\hline 1 & $\mathrm{H}$ & 3.214533 & -3.023135 & 0.907031 \\
\hline 2 & $\mathrm{C}$ & 2.773802 & -2.070798 & 0.622549 \\
\hline 3 & $\mathrm{H}$ & 4.579850 & -0.960633 & 0.866714 \\
\hline 4 & $\mathrm{C}$ & 3.528873 & -0.938941 & 0.589450 \\
\hline 5 & $\mathrm{C}$ & 0.730271 & -0.812798 & 0.012833 \\
\hline 6 & $\mathrm{C}$ & 2.975237 & 0.278198 & 0.083821 \\
\hline 7 & $\mathrm{C}$ & 1.382110 & -2.047151 & 0.289266 \\
\hline 8 & $\mathrm{C}$ & 1.588099 & 0.337389 & -0.261679 \\
\hline 9 & $\mathrm{C}$ & 3.811794 & 1.395240 & -0.160761 \\
\hline 10 & $\mathrm{H}$ & 1.197308 & -4.203121 & 0.383635 \\
\hline 11 & $\mathrm{C}$ & -1.365498 & -2.060426 & -0.313520 \\
\hline 12 & $\mathrm{C}$ & -0.642897 & -3.281313 & -0.192688 \\
\hline 13 & $\mathrm{C}$ & 3.333844 & 2.516836 & -0.803672 \\
\hline 14 & $\mathrm{H}$ & 4.855336 & 1.334531 & 0.139323 \\
\hline 15 & $\mathrm{H}$ & 3.989038 & 3.361486 & -0.998044 \\
\hline 16 & C & 1.997046 & 2.538926 & -1.247754 \\
\hline 17 & $\mathrm{H}$ & 1.627206 & 3.390710 & -1.812076 \\
\hline 18 & $\mathrm{C}$ & 1.151790 & 1.479079 & -0.983683 \\
\hline 19 & $\mathrm{H}$ & -1.171393 & -4.217239 & -0.355312 \\
\hline 20 & C & -0.719004 & -0.816901 & -0.067294 \\
\hline 21 & $\mathrm{C}$ & -3.517006 & -0.969134 & -0.640517 \\
\hline 22 & C & -1.581800 & 0.335926 & 0.179142 \\
\hline 23 & C & -2.757057 & -2.098194 & -0.646054 \\
\hline 24 & C & -2.968651 & 0.262346 & -0.164740 \\
\hline 25 & $\mathrm{C}$ & -1.150457 & 1.496748 & 0.873077 \\
\hline 26 & $\mathrm{H}$ & -3.193661 & -3.059075 & -0.907212 \\
\hline 27 & $\mathrm{H}$ & -4.853286 & 1.308893 & -0.245877 \\
\hline 28 & $\mathrm{H}$ & -4.567864 & -1.002111 & -0.917115 \\
\hline 29 & $\mathrm{C}$ & -2.000291 & 2.559062 & 1.111276 \\
\hline 30 & $\mathrm{H}$ & -0.135114 & 1.544914 & 1.244753 \\
\hline 31 & $\mathrm{H}$ & -1.634158 & 3.425938 & 1.654647 \\
\hline 32 & $\mathrm{C}$ & -3.336957 & 2.520386 & 0.667936 \\
\hline 33 & $\mathrm{H}$ & -3.995799 & 3.366690 & 0.841693 \\
\hline 34 & $\mathrm{C}$ & -3.810031 & 1.381400 & 0.052583 \\
\hline 35 & C & 0.664789 & -3.273727 & 0.198271 \\
\hline
\end{tabular}

Energy is $\quad-846.1203278643$

\footnotetext{
$\S$ Radical 3 is not an energy minimum; it isomerizes spontaneously to the cyclized radical. The energy calculated here was obtained on the radical generated by removal of a hydrogen from $\mathbf{1}$ with all the other atoms frozen.
} 
Calculated DFT (UB3LYP/6-31G*) energy and X,Y,Z-coordinates for carbene 4

\begin{tabular}{|c|c|c|c|c|}
\hline & & Coor & lates & coms ) \\
\hline & ГOM & $\mathrm{X}$ & $\mathrm{Y}$ & $\mathrm{Z}$ \\
\hline 1 & $\mathrm{C}$ & 0.800013 & -0.918631 & 0.022892 \\
\hline 2 & $\mathrm{H}$ & -1.003355 & -4.402413 & 0.279532 \\
\hline 3 & $\mathrm{C}$ & -0.640527 & -0.983933 & 0.036424 \\
\hline 4 & $\mathrm{C}$ & -1.270934 & -2.251354 & 0.196278 \\
\hline 5 & $\mathrm{C}$ & 1.516709 & -2.135295 & -0.162389 \\
\hline 6 & $\mathrm{C}$ & -1.045282 & 1.268550 & -0.972673 \\
\hline 7 & $\mathrm{C}$ & 0.843466 & -3.388684 & -0.080521 \\
\hline 8 & $\mathrm{C}$ & 2.919538 & -2.110270 & -0.427893 \\
\hline 9 & $\mathrm{C}$ & -0.497267 & -3.447407 & 0.162661 \\
\hline 10 & $\mathrm{H}$ & 1.429303 & -4.298320 & -0.185979 \\
\hline 11 & $\mathrm{C}$ & -1.503303 & 0.167956 & -0.208200 \\
\hline 12 & $\mathrm{C}$ & -2.686888 & -2.327689 & 0.334729 \\
\hline 13 & $\mathrm{C}$ & 3.609754 & -0.935891 & -0.458530 \\
\hline 14 & $\mathrm{H}$ & 3.423364 & -3.054089 & -0.621156 \\
\hline 15 & $\mathrm{C}$ & 2.974168 & 0.290523 & -0.096713 \\
\hline 16 & $\mathrm{H}$ & 4.671396 & -0.922432 & -0.693186 \\
\hline 17 & $\mathrm{C}$ & 1.576040 & 0.302982 & 0.204405 \\
\hline 18 & $\mathrm{C}$ & 3.738586 & 1.477432 & 0.038854 \\
\hline 19 & $\mathrm{C}$ & -3.731457 & 1.252970 & 0.063159 \\
\hline 20 & $\mathrm{C}$ & 1.054083 & 1.491587 & 0.783804 \\
\hline 21 & $\mathrm{C}$ & -3.483117 & -1.210368 & 0.300296 \\
\hline 22 & $\mathrm{H}$ & -3.132660 & -3.309886 & 0.475685 \\
\hline 23 & $\mathrm{C}$ & -2.908809 & 0.056729 & 0.042410 \\
\hline 24 & $\mathrm{H}$ & -4.556556 & -1.293267 & 0.454641 \\
\hline 25 & $\mathrm{C}$ & 3.178375 & 2.630870 & 0.541193 \\
\hline 26 & $\mathrm{H}$ & 4.791444 & 1.447364 & -0.231484 \\
\hline 27 & $\mathrm{C}$ & 1.831175 & 2.620280 & 0.954386 \\
\hline 28 & $\mathrm{H}$ & 3.778578 & 3.529355 & 0.656394 \\
\hline 29 & $\mathrm{C}$ & -1.928157 & 2.469360 & -1.052423 \\
\hline 30 & $\mathrm{H}$ & 1.400911 & 3.502267 & 1.421073 \\
\hline 31 & $\mathrm{H}$ & -4.726618 & 1.163371 & 0.493673 \\
\hline 32 & $\mathrm{C}$ & -3.263281 & 2.435447 & -0.376550 \\
\hline 33 & $\mathrm{H}$ & -3.833033 & 3.353879 & -0.246721 \\
\hline 34 & $\mathrm{H}$ & -1.320927 & 3.321735 & -0.693984 \\
\hline 35 & $\mathrm{H}$ & -2.046920 & 2.712430 & -2.123386 \\
\hline 36 & $\mathrm{H}$ & 0.042189 & 1.494839 & 1.169298 \\
\hline
\end{tabular}

Energy is $\quad-846.675061688$ 
Calculated DFT (UB3LYP/6-31G*) energy and X,Y,Z-coordinates for intermediate 5

\begin{tabular}{|c|c|c|c|c|}
\hline & & Coorc & nates & coms ) \\
\hline & ГОМ & $\mathrm{X}$ & $\mathrm{Y}$ & $\mathrm{Z}$ \\
\hline 1 & $\mathrm{C}$ & 0.729363 & 0.022838 & 0.997312 \\
\hline 2 & $\mathrm{H}$ & -1.234438 & -0.032275 & 4.396527 \\
\hline 3 & $\mathrm{C}$ & -0.729363 & -0.022838 & 0.997312 \\
\hline 4 & $\mathrm{C}$ & -1.429173 & -0.043590 & 2.260585 \\
\hline 5 & $\mathrm{C}$ & 1.429173 & 0.043590 & 2.260585 \\
\hline 6 & $\mathrm{C}$ & -0.712980 & -0.278398 & -1.513705 \\
\hline 7 & $\mathrm{C}$ & 0.698222 & 0.018182 & 3.450633 \\
\hline 8 & $\mathrm{C}$ & 2.859924 & 0.111855 & 2.247406 \\
\hline 9 & $\mathrm{C}$ & -0.698222 & -0.018182 & 3.450633 \\
\hline 10 & $\mathrm{H}$ & 1.234438 & 0.032275 & 4.396527 \\
\hline 11 & $\mathrm{C}$ & -1.437924 & -0.082541 & -0.193297 \\
\hline 12 & $\mathrm{C}$ & -2.859924 & -0.111855 & 2.247406 \\
\hline 13 & $\mathrm{C}$ & 3.553894 & 0.137679 & 1.076233 \\
\hline 14 & $\mathrm{H}$ & 3.383694 & 0.139883 & 3.200030 \\
\hline 15 & $\mathrm{C}$ & 2.873989 & 0.101154 & -0.190860 \\
\hline 16 & $\mathrm{H}$ & 4.640701 & 0.173795 & 1.077930 \\
\hline 17 & $\mathrm{C}$ & 1.437924 & 0.082541 & -0.193297 \\
\hline 18 & $\mathrm{C}$ & 3.566177 & -0.006655 & -1.395526 \\
\hline 19 & $\mathrm{C}$ & -3.566177 & 0.006655 & -1.395526 \\
\hline 20 & $\mathrm{C}$ & 0.712980 & 0.278398 & -1.513705 \\
\hline 21 & $\mathrm{C}$ & -3.553894 & -0.137679 & 1.076233 \\
\hline 22 & $\mathrm{H}$ & -3.383694 & -0.139883 & 3.200030 \\
\hline 23 & $\mathrm{C}$ & -2.873989 & -0.101154 & -0.190860 \\
\hline 24 & $\mathrm{H}$ & -4.640701 & -0.173795 & 1.077930 \\
\hline 25 & $\mathrm{C}$ & 2.877267 & -0.264947 & -2.618660 \\
\hline 26 & $\mathrm{H}$ & 4.652995 & -0.014512 & -1.381859 \\
\hline 27 & $\mathrm{C}$ & 1.521515 & -0.228803 & -2.679941 \\
\hline 28 & $\mathrm{H}$ & 3.457487 & -0.546759 & -3.494429 \\
\hline 29 & $\mathrm{H}$ & 0.617407 & 1.387741 & -1.641512 \\
\hline 30 & $\mathrm{H}$ & 1.003469 & -0.494357 & -3.596866 \\
\hline 31 & $\mathrm{H}$ & -4.652995 & 0.014512 & -1.381859 \\
\hline 32 & $\mathrm{C}$ & -2.877267 & 0.264947 & -2.618660 \\
\hline 33 & $\mathrm{H}$ & -3.457487 & 0.546759 & -3.494429 \\
\hline 34 & $\mathrm{H}$ & -1.003469 & 0.494357 & -3.596866 \\
\hline 35 & $\mathrm{C}$ & -1.521515 & 0.228803 & -2.679941 \\
\hline 36 & $\mathrm{H}$ & -0.617407 & -1.387741 & -1.641512 \\
\hline
\end{tabular}

Energy is $\quad-846.730145604$ 
Calculated DFT (UB3LYP/6-31G*) energy and X,Y,Z-coordinates for benzo[5]helicene 6

\begin{tabular}{|c|c|c|c|c|}
\hline & & Coore & lates & coms ) \\
\hline & ГOM & $\mathrm{X}$ & $\mathrm{Y}$ & $\mathrm{Z}$ \\
\hline 1 & $\mathrm{C}$ & 0.023603 & -0.730640 & -0.061747 \\
\hline 2 & $\mathrm{C}$ & -0.492543 & 1.296713 & -3.779042 \\
\hline 3 & $\mathrm{C}$ & -0.023603 & 0.730640 & -0.061747 \\
\hline 4 & $\mathrm{C}$ & -0.324400 & 1.392560 & -1.270828 \\
\hline 5 & $\mathrm{C}$ & 0.324400 & -1.392560 & -1.270828 \\
\hline 6 & $\mathrm{C}$ & 1.045726 & 1.047519 & 2.210325 \\
\hline 7 & $\mathrm{C}$ & 0.234876 & -0.671610 & -2.537142 \\
\hline 8 & $\mathrm{C}$ & 0.637169 & -2.786770 & -1.236545 \\
\hline 9 & $\mathrm{C}$ & -0.234876 & 0.671610 & -2.537142 \\
\hline 10 & $\mathrm{H}$ & 0.871734 & -2.311253 & -3.806815 \\
\hline 11 & $\mathrm{C}$ & 0.310152 & 1.542449 & 1.098923 \\
\hline 12 & $\mathrm{C}$ & -0.637169 & 2.786770 & -1.236545 \\
\hline 13 & $\mathrm{C}$ & 0.543214 & -3.517557 & -0.087813 \\
\hline 14 & $\mathrm{H}$ & 0.959772 & -3.285311 & -2.142473 \\
\hline 15 & $\mathrm{C}$ & -0.000078 & -2.937759 & 1.092784 \\
\hline 16 & $\mathrm{H}$ & 0.808913 & -4.571797 & -0.082662 \\
\hline 17 & $\mathrm{C}$ & -0.310152 & -1.542449 & 1.098923 \\
\hline 18 & $\mathrm{C}$ & -0.302905 & -3.737073 & 2.224330 \\
\hline 19 & $\mathrm{C}$ & 0.302905 & 3.737073 & 2.224330 \\
\hline 20 & $\mathrm{C}$ & -1.045726 & -1.047519 & 2.210325 \\
\hline 21 & $\mathrm{C}$ & -0.543214 & 3.517557 & -0.087813 \\
\hline 22 & $\mathrm{H}$ & -0.959772 & 3.285311 & -2.142473 \\
\hline 23 & $\mathrm{C}$ & 0.000078 & 2.937759 & 1.092784 \\
\hline 24 & $\mathrm{H}$ & -0.808913 & 4.571797 & -0.082662 \\
\hline 25 & $\mathrm{C}$ & -0.964558 & -3.207479 & 3.309484 \\
\hline 26 & $\mathrm{H}$ & -0.029641 & -4.789452 & 2.202484 \\
\hline 27 & $\mathrm{C}$ & -1.366007 & -1.855277 & 3.282048 \\
\hline 28 & $\mathrm{H}$ & -1.204916 & -3.830817 & 4.166258 \\
\hline 29 & $\mathrm{C}$ & 1.366007 & 1.855277 & 3.282048 \\
\hline 30 & $\mathrm{H}$ & -1.941362 & -1.445585 & 4.107766 \\
\hline 31 & $\mathrm{H}$ & 0.029641 & 4.789452 & 2.202484 \\
\hline 32 & $\mathrm{C}$ & 0.964558 & 3.207479 & 3.309484 \\
\hline 33 & $\mathrm{H}$ & 1.204916 & 3.830817 & 4.166258 \\
\hline 34 & $\mathrm{H}$ & 1.941362 & 1.445585 & 4.107766 \\
\hline 35 & $\mathrm{H}$ & 1.385079 & 0.019776 & 2.207401 \\
\hline 36 & $\mathrm{H}$ & -1.385079 & -0.019776 & 2.207401 \\
\hline 37 & C & -0.257535 & 0.652051 & -4.979552 \\
\hline 38 & $\mathrm{H}$ & -0.871734 & 2.311253 & -3.806815 \\
\hline 39 & $\mathrm{H}$ & -0.462002 & 1.161329 & -5.917185 \\
\hline 40 & $\mathrm{C}$ & 0.257535 & -0.652051 & -4.979552 \\
\hline 41 & $\mathrm{C}$ & 0.492543 & -1.296713 & -3.779042 \\
\hline 42 & $\mathrm{H}$ & 0.462002 & -1.161329 & -5.917185 \\
\hline
\end{tabular}

Energy is $\quad-1000.447846586$ 
Calculated DFT (UB3LYP/6-31G*) energy and X,Y,Z-coordinates for intermediate 7

\begin{tabular}{|c|c|c|c|c|}
\hline & & Coord & lates & coms ) \\
\hline & ГOM & $\mathrm{x}$ & $\mathrm{Y}$ & $\mathrm{Z}$ \\
\hline 1 & $\mathrm{C}$ & -0.026636 & -0.732891 & -0.167298 \\
\hline 2 & $\mathrm{C}$ & 0.009731 & 1.389630 & -3.912838 \\
\hline 3 & $\mathrm{C}$ & 0.026636 & 0.732891 & -0.167298 \\
\hline 4 & $\mathrm{C}$ & 0.048540 & 1.441917 & -1.414518 \\
\hline 5 & $\mathrm{C}$ & -0.048540 & -1.441917 & -1.414518 \\
\hline 6 & $\mathrm{C}$ & 0.264720 & 0.713758 & 2.359390 \\
\hline 7 & $\mathrm{C}$ & -0.012981 & -0.718227 & -2.654877 \\
\hline 8 & $\mathrm{C}$ & -0.129469 & -2.864983 & -1.370521 \\
\hline 9 & $\mathrm{C}$ & 0.012981 & 0.718227 & -2.654877 \\
\hline 10 & $\mathrm{H}$ & -0.010480 & -2.472296 & -3.942545 \\
\hline 11 & $\mathrm{C}$ & 0.081502 & 1.435969 & 1.038930 \\
\hline 12 & C & 0.129469 & 2.864983 & -1.370521 \\
\hline 13 & $\mathrm{C}$ & -0.154317 & -3.551162 & -0.189597 \\
\hline 14 & $\mathrm{H}$ & -0.174411 & -3.430933 & -2.292897 \\
\hline 15 & $\mathrm{C}$ & -0.106774 & -2.864126 & 1.059466 \\
\hline 16 & $\mathrm{H}$ & -0.199801 & -4.637523 & -0.188476 \\
\hline 17 & $\mathrm{C}$ & -0.081502 & -1.435969 & 1.038930 \\
\hline 18 & $\mathrm{C}$ & 0.004467 & -3.554268 & 2.278173 \\
\hline 19 & $\mathrm{C}$ & -0.004467 & 3.554268 & 2.278173 \\
\hline 20 & $\mathrm{C}$ & -0.264720 & -0.713758 & 2.359390 \\
\hline 21 & $\mathrm{C}$ & 0.154317 & 3.551162 & -0.189597 \\
\hline 22 & $\mathrm{H}$ & 0.174411 & 3.430933 & -2.292897 \\
\hline 23 & $\mathrm{C}$ & 0.106774 & 2.864126 & 1.059466 \\
\hline 24 & $\mathrm{H}$ & 0.199801 & 4.637523 & -0.188476 \\
\hline 25 & $\mathrm{C}$ & 0.294961 & -2.863549 & 3.481441 \\
\hline 26 & $\mathrm{H}$ & 0.000523 & -4.640999 & 2.266634 \\
\hline 27 & $\mathrm{C}$ & 0.279860 & -1.501257 & 3.519032 \\
\hline 28 & $\mathrm{H}$ & 0.591367 & -3.434592 & 4.358453 \\
\hline 29 & $\mathrm{H}$ & -1.375481 & -0.635779 & 2.506100 \\
\hline 30 & $\mathrm{H}$ & 0.586280 & -0.967078 & 4.413415 \\
\hline 31 & $\mathrm{H}$ & -0.000523 & 4.640999 & 2.266634 \\
\hline 32 & $\mathrm{C}$ & -0.294961 & 2.863549 & 3.481441 \\
\hline 33 & $\mathrm{H}$ & -0.591367 & 3.434592 & 4.358453 \\
\hline 34 & $\mathrm{H}$ & -0.586280 & 0.967078 & 4.413415 \\
\hline 35 & $\mathrm{C}$ & -0.279860 & 1.501257 & 3.519032 \\
\hline 36 & $\mathrm{H}$ & 1.375481 & 0.635779 & 2.506100 \\
\hline 37 & $\mathrm{C}$ & 0.001537 & 0.706562 & -5.103950 \\
\hline 38 & $\mathrm{H}$ & 0.010480 & 2.472296 & -3.942545 \\
\hline 39 & $\mathrm{H}$ & -0.000047 & 1.252110 & -6.043515 \\
\hline 40 & $\mathrm{C}$ & -0.001537 & -0.706562 & -5.103950 \\
\hline 41 & $\mathrm{C}$ & -0.009731 & -1.389630 & -3.912838 \\
\hline 42 & $\mathrm{H}$ & 0.000047 & -1.252110 & -6.043515 \\
\hline
\end{tabular}

Energy is $\quad-1000.363221585$ 
Calculated DFT (UB3LYP/6-31G*) energy and X,Y,Z-coordinates for the benzannulated derivative of radical $3^{\S}$

\begin{tabular}{|c|c|c|c|c|}
\hline & & Coorc & tes (Angst & ns ) \\
\hline & АТОМ & $\mathrm{X}$ & $\mathrm{Y}$ & Z \\
\hline 1 & $\mathrm{H}$ & 2.094698 & -3.276425 & -1.008948 \\
\hline 2 & $\mathrm{C}$ & 1.186227 & -2.780264 & -0.689657 \\
\hline 3 & $\mathrm{H}$ & 0.032529 & -4.562282 & -0.891236 \\
\hline 4 & $\mathrm{C}$ & 0.035998 & -3.510881 & -0.614357 \\
\hline 5 & $\mathrm{C}$ & 0.007589 & -0.729512 & -0.065275 \\
\hline 6 & $\mathrm{C}$ & -1.149111 & -2.935656 & -0.076096 \\
\hline 7 & $\mathrm{C}$ & 1.218865 & -1.389454 & -0.361727 \\
\hline 8 & $\mathrm{C}$ & -1.156978 & -1.543659 & 0.248663 \\
\hline 9 & $\mathrm{C}$ & -2.284114 & -3.736950 & 0.207510 \\
\hline 10 & $\mathrm{C}$ & 2.484999 & -0.670874 & -0.252445 \\
\hline 11 & $\mathrm{H}$ & -2.274404 & -0.031109 & 1.328757 \\
\hline 12 & $\mathrm{C}$ & 1.179685 & 2.779319 & 0.644680 \\
\hline 13 & $\mathrm{C}$ & -3.375073 & -3.213165 & 0.864369 \\
\hline 14 & $\mathrm{H}$ & -2.260627 & -4.786473 & -0.076491 \\
\hline 15 & $\mathrm{H}$ & -4.234636 & -3.838250 & 1.090031 \\
\hline 16 & $\mathrm{C}$ & -3.350455 & -1.865236 & 1.280221 \\
\hline 17 & $\mathrm{H}$ & -4.181319 & -1.460754 & 1.851898 \\
\hline 18 & $\mathrm{C}$ & -2.274957 & -1.055352 & 0.978803 \\
\hline 19 & $\mathrm{H}$ & 0.026314 & 4.563898 & 0.824275 \\
\hline 20 & $\mathrm{C}$ & 0.032725 & 3.512486 & 0.547490 \\
\hline 21 & $\mathrm{C}$ & 0.008608 & 0.731176 & -0.002092 \\
\hline 22 & $\mathrm{C}$ & -1.143186 & 2.939882 & -0.013268 \\
\hline 23 & $\mathrm{C}$ & 1.215477 & 1.388433 & 0.317402 \\
\hline 24 & $\mathrm{C}$ & -1.147954 & 1.547900 & -0.338148 \\
\hline 25 & $\mathrm{C}$ & -2.270797 & 3.743689 & -0.318435 \\
\hline 26 & $\mathrm{H}$ & 3.747026 & 2.297899 & 0.900014 \\
\hline 27 & $\mathrm{H}$ & 5.866537 & -1.161643 & -0.453352 \\
\hline 28 & $\mathrm{H}$ & 2.083006 & 3.273468 & 0.981236 \\
\hline 29 & $\mathrm{C}$ & -3.350201 & 3.222314 & -0.995975 \\
\hline 30 & $\mathrm{H}$ & -2.250396 & 4.793163 & -0.034015 \\
\hline 31 & $\mathrm{H}$ & -4.203917 & 3.849301 & -1.237962 \\
\hline 32 & $\mathrm{C}$ & -3.320656 & 1.874324 & -1.411311 \\
\hline 33 & $\mathrm{H}$ & -4.141371 & 1.471676 & -1.998726 \\
\hline 34 & $\mathrm{C}$ & -2.252898 & 1.062061 & -1.089470 \\
\hline 35 & $\mathrm{C}$ & 4.923879 & 0.644447 & 0.278199 \\
\hline 36 & $\mathrm{C}$ & 2.481866 & 0.667041 & 0.232253 \\
\hline 37 & $\mathrm{C}$ & 3.728631 & -1.294332 & -0.505546 \\
\hline 38 & $\mathrm{C}$ & 4.927489 & -0.653703 & -0.251847 \\
\hline 39 & $\mathrm{C}$ & 3.721831 & 1.287742 & 0.509019 \\
\hline 40 & $\mathrm{H}$ & 5.860042 & 1.150305 & 0.497572 \\
\hline 41 & $\mathrm{H}$ & 3.759030 & -2.304551 & -0.896011 \\
\hline
\end{tabular}

Energy is $\quad-999.7609290154$

$\S$ This radical, like 3, is not an energy minimum; it isomerizes spontaneously to the cyclized radical. The energy calculated here was obtained on the radical generated by removal of a hydrogen from $\mathbf{6}$ with all the other atoms frozen. 
Calculated DFT (UB3LYP/6-31G*) energy and X,Y,Z-coordinates for the benzannulated derivative of carbene 4

\begin{tabular}{|c|c|c|c|c|}
\hline \multirow{2}{*}{\multicolumn{2}{|c|}{ АTOM }} & \multicolumn{3}{|c|}{ Coordinates (Angstroms) } \\
\hline & & $\mathrm{x}$ & $\mathrm{Y}$ & $\mathrm{z}$ \\
\hline 1 & $\mathrm{C}$ & -0.182828 & -0.735470 & 0.005477 \\
\hline 2 & $\mathrm{C}$ & -3.814569 & 1.485709 & 0.389684 \\
\hline 3 & $\mathrm{C}$ & -0.122181 & 0.712858 & 0.007637 \\
\hline 4 & $\mathrm{C}$ & -1.309122 & 1.467316 & 0.155927 \\
\hline 5 & $\mathrm{C}$ & -1.420333 & -1.352952 & -0.282801 \\
\hline 6 & $\mathrm{C}$ & 2.163255 & 0.787767 & -0.993658 \\
\hline 7 & $\mathrm{C}$ & -2.659456 & -0.586781 & -0.177698 \\
\hline 8 & C & -1.431592 & -2.734600 & -0.635031 \\
\hline 9 & $\mathrm{C}$ & -2.605007 & 0.796185 & 0.148578 \\
\hline 10 & $\mathrm{H}$ & -3.998993 & -2.254045 & -0.538001 \\
\hline 11 & $\mathrm{C}$ & 1.121910 & 1.415168 & -0.262189 \\
\hline 12 & C & -1.210381 & 2.882336 & 0.234900 \\
\hline 13 & $\mathrm{C}$ & -0.293111 & -3.490688 & -0.606153 \\
\hline 14 & $\mathrm{H}$ & -2.354925 & -3.198565 & -0.960472 \\
\hline 15 & $\mathrm{C}$ & 0.922197 & -2.958734 & -0.095642 \\
\hline 16 & $\mathrm{H}$ & -0.321360 & -4.533872 & -0.911533 \\
\hline 17 & $\mathrm{C}$ & 0.975264 & -1.576306 & 0.261985 \\
\hline 18 & $\mathrm{C}$ & 2.050018 & -3.792626 & 0.122317 \\
\hline 19 & C & 2.474424 & 3.501174 & -0.057502 \\
\hline 20 & C & 2.123959 & -1.138650 & 0.982031 \\
\hline 21 & $\mathrm{C}$ & -0.004123 & 3.545385 & 0.175189 \\
\hline 22 & $\mathrm{H}$ & -2.112981 & 3.472761 & 0.342998 \\
\hline 23 & C & 1.185965 & 2.830647 & -0.057866 \\
\hline 24 & $\mathrm{H}$ & 0.028048 & 4.626487 & 0.291824 \\
\hline 25 & $\mathrm{C}$ & 3.170083 & -3.314951 & 0.762727 \\
\hline 26 & $\mathrm{H}$ & 1.995074 & -4.830855 & -0.196432 \\
\hline 27 & C & 3.185295 & -1.983348 & 1.231638 \\
\hline 28 & $\mathrm{H}$ & 4.021312 & -3.965970 & 0.942297 \\
\hline 29 & $\mathrm{C}$ & 3.468464 & 1.507170 & -1.072441 \\
\hline 30 & $\mathrm{H}$ & 4.035692 & -1.622036 & 1.803848 \\
\hline 31 & $\mathrm{H}$ & 2.505394 & 4.519274 & 0.325734 \\
\hline 32 & C & 3.592105 & 2.867201 & -0.456421 \\
\hline 33 & $\mathrm{H}$ & 4.573131 & 3.325412 & -0.343300 \\
\hline 34 & $\mathrm{H}$ & 4.223144 & 0.811814 & -0.659508 \\
\hline 35 & $\mathrm{H}$ & 3.756139 & 1.544805 & -2.138066 \\
\hline 36 & $\mathrm{H}$ & 2.144672 & -0.137348 & 1.394294 \\
\hline 37 & $\mathrm{C}$ & -5.041595 & 0.858103 & 0.276941 \\
\hline 38 & $\mathrm{H}$ & -3.793682 & 2.530516 & 0.678114 \\
\hline 39 & $\mathrm{H}$ & -5.955016 & 1.414206 & 0.468530 \\
\hline 40 & C & -5.098704 & -0.496104 & -0.082900 \\
\hline 41 & $\mathrm{C}$ & -3.928171 & -1.199734 & -0.298734 \\
\hline 42 & $\mathrm{H}$ & -6.057414 & -0.998661 & -0.176323 \\
\hline
\end{tabular}

Energy is -1000.316265899 